\title{
Superiority of Cystatin C over Creatinine for Early Diagnosis of Acute Kidney Injury in Pediatric Acute Lymphoblastic Leukemia/ Lymphoblastic Lymphoma
}

\author{
Takashi Yamazoe, ${ }^{1, *}$ Shohei Akagawa, ${ }^{1, *}$ Ryosuke Matsuno, ${ }^{1}$ Yuko Akagawa, ${ }^{1}$ \\ Sohsaku Yamanouchi, ${ }^{1}$ Taichi Omachi, ${ }^{1}$ Takahisa Kimata, ${ }^{1}$ Shoji Tsuji ${ }^{1}$ and \\ Kazunari Kaneko ${ }^{1}$ \\ ${ }^{1}$ Department of Pediatrics, Kansai Medical University, Hirakata, Osaka, Japan
}

\begin{abstract}
The exact incidence of acute kidney injury (AKI) during chemotherapy for acute lymphoblastic leukemia (ALL)/lymphoblastic lymphoma (LBL) is unknown. Furthermore, childhood cancer survivors are at risk of AKI-chronic kidney disease transition. Thus, early diagnosis of AKI is crucial. This study aimed to elucidate the incidence of $A K I$ in patients undergoing chemotherapy for pediatric ALL/LBL and to compare the usefulness of serum cystatin $C$ (CysC)- and creatinine ( $\mathrm{Cr}$ )-based estimated glomerular filtration rate (eGFR) as diagnostic measures. Data of 16 patients with ALL/LBL treated with a total of 75 courses of chemotherapy were retrospectively analyzed. CysC- and Cr-based eGFR were measured before and three times per week during therapy. To calculate the eGFR, an equation for Japanese children was used. AKI was diagnosed when eGFR dropped by $\geq 25 \%$ from the highest eGFR value obtained during the latest 2 weeks since the start of chemotherapy. AKI was graded based on the pediatric Risk, Injury, Failure, Loss, End Stage Renal Disease scale. All patients developed AKI during chemotherapy; however, more than $90 \%$ of the cases were mild and eventually recovered. No significant differences were found in the incidence of AKI between CysC- and Cr-based eGFR ( $p=0.104)$. The median time to AKI diagnosis was significantly shorter in the CysC-based eGFR than in the Cr-based eGFR ( 8 vs. 17 days, $p<0.001$ ). In this study, all patients with pediatric ALL/LBL could develop mild AKI during treatment. CysC-based eGFR is a more effective measure than Cr-based eGFR for the early diagnosis of AKI.
\end{abstract}

Keywords: acute kidney injury; acute lymphoblastic leukemia; chemotherapy; creatinine; cystatin C Tohoku J. Exp. Med., 2021 July, 254 (3), 163-170.

\section{Introduction}

Acute kidney injury (AKI) is one of the serious comorbidities associated with the treatment of leukemia as it increases the mortality rate. In adult leukemia (acute myeloid leukemia and high-risk myelodysplastic syndrome), one-third of the patients undergoing chemotherapy develop AKI, and these patients demonstrate a five-fold increase in the mortality rate (Lahoti et al. 2010). However, in both adult and childhood acute lymphoblastic leukemia (ALL), the incidence of AKI during chemotherapy is unknown except for those related to the tumor lysis syndrome or associated with specific chemotherapeutic regi- mens such as methotrexate (Perazella 1999), cisplatin (Pabla and Dong 2008), and ifosfamide (Ciarimboli et al. 2011).

AKI is a risk factor for the future development of chronic kidney disease (CKD), a condition characterized by progressive loss of renal function. According to previous reports from the Childhood Cancer Survivor Study, the prevalence of CKD ranges from $2.4 \%$ to $32 \%$ among childhood cancer survivors (Kooijmans et al. 2019). Thus, early diagnosis and treatment of AKI during chemotherapy for ALL, which could reduce the risk of AKI-CKD transition, has clinical significance.

Although serum creatinine $(\mathrm{Cr})$ is broadly used for the

\footnotetext{
Received February 19, 2021; revised and accepted May 5, 2021. Published online July 9, 2021; doi: 10.1620/tjem.254.163.

*These two authors contributed equally to this work.

Correspondence: Kazunari Kaneko, M.D., Ph.D., Department of Pediatrics, Kansai Medical University, 2-5-1 Shin-machi, Hirakata, Osaka 573-1010, Japan.

e-mail:kanekok@hirakata.kmu.ac.jp

(C)2021 Tohoku University Medical Press. This is an open-access article distributed under the terms of the Creative Commons Attribution-NonCommercial-NoDerivatives 4.0 International License (CC-BY-NC-ND 4.0). Anyone may download, reuse, copy, reprint, or distribute the article without modifications or adaptations for non-profit purposes if they cite the original authors and source properly.

https://creativecommons.org/licenses/by-nc-nd/4.0/
} 
diagnosis of AKI, it is affected by several factors other than renal function, including sex, age, and muscle mass (Kellum et al. 2013). Cystatin C (CysC), which is not affected by the above-mentioned factors (Laterza et al. 2002), could potentially be more suitable and sensitive for the assessment of renal function in children (Lankisch et al. 2006; Nakhjavan-Shahraki et al. 2017).

In this study, we aimed to elucidate the incidence of AKI in pediatric ALL/lymphoblastic lymphoma (LBL) during chemotherapy and to compare the efficacy of the CysCbased estimated glomerular filtration rate (eGFR) with the Cr-based eGFR in the diagnosis of AKI during ALL/LBL treatment.

\section{Materials and Methods}

\section{Ethics approval and consent to participate}

All procedures performed in this study were in accordance with the ethical standards of the institutional research committee at which the studies were conducted (Kansai Medical University; no. 2019288) and with the 1964 Helsinki declaration and its later amendments or comparable ethical standards. The authors did not conduct experiments on animals in this study.

\section{Participants and sample collection}

Fourteen ALL patient and two LBL patients aged 2-18 years treated at the Pediatric Department of Kansai Medical University Hospital from February 2016 to July 2020 were included. No participants showed elevated $\mathrm{Cr}$ or CysC due to tumor lysis syndrome or blast infiltration at diagnosis. The patients received a total of 75 courses of chemotherapy. Serum $\mathrm{CysC}$ and $\mathrm{Cr}$ levels were measured to calculate eGFR (CysC-based eGFR and Cr-based eGFR, respectively) (Uemura et al. 2014a, b), before and three times a week during each course of chemotherapy. There were 14 courses of induction, 12 courses of early consolidation, 25 courses of consolidation, and 24 courses of re-induction (Table 1). These chemotherapies included cyclophosphamide, cytarabine, dasatinib, daunorubicin, doxorubicin, etoposide, ifosfamide, L-asparaginase, mercaptopurine, methotrexate, pirarubicin, vincristine, and vindesine. The patients were treated according to the Japanese Pediatric Leukemia/ Lymphoma Study Group protocol.

\section{Assessment of AKI}

AKI was diagnosed when eGFR dropped by $25 \%$ or more from the highest eGFR value obtained during the latest 2 weeks since the start of chemotherapy. AKI was graded based on the pediatric Risk, Injury, Failure, Loss, End Stage Renal Disease scale (pRIFLE) (Akcan-Arikan et al. 2007) as shown in Table 2, though the reduction in urine output was not applied in this study. AKI was diagnosed

Table 1. Patient clinical characteristics and incidence of acute kidney injury (AKI).

\begin{tabular}{|c|c|c|c|c|c|c|c|c|c|c|c|c|c|}
\hline \multirow{2}{*}{ Case } & \multirow{2}{*}{ Sex } & \multirow{2}{*}{ Age, year } & \multirow{2}{*}{$\begin{array}{l}\text { Weight } \\
\text { Z score }\end{array}$} & \multirow{2}{*}{ BMI } & \multirow{2}{*}{$\begin{array}{c}\text { Type of } \\
\text { leukemia }\end{array}$} & \multirow{2}{*}{ Risk/stage } & \multicolumn{4}{|c|}{ Courses of chemotherapy } & \multirow{2}{*}{$\begin{array}{c}{ }^{*} \text { CysC- } \\
- \text { based } \\
\text { eGFR } \\
\text { AKI }\end{array}$} & \multirow{2}{*}{$\begin{array}{c}{ }^{\dagger} \mathrm{Cr}- \\
\text { based } \\
\text { eGFR } \\
\text { AKI }\end{array}$} & \multirow{2}{*}{$\begin{array}{c}\text { AKI by } \\
\text { both }\end{array}$} \\
\hline & & & & & & & I & $\mathrm{EC}$ & $\mathrm{C}$ & $\mathrm{R}$ & & & \\
\hline 1 & M & 2.0 & -1.3 & 15.8 & ALL & Intermediate/(-) & 1 & 1 & 1 & 2 & 3 & 4 & 3 \\
\hline 2 & M & 2.9 & -0.4 & 16.3 & ALL & Standard/(-) & & & & 1 & 1 & 1 & 1 \\
\hline 3 & M & 4.2 & 0.8 & 15.4 & ALL & Standard $/(-)$ & 1 & 1 & 1 & 2 & 3 & 2 & 1 \\
\hline 4 & M & 6.2 & -0.8 & 14.7 & ALL & $\mathrm{Ph}$ positive/(-) & 1 & 2 & 3 & 2 & 8 & 6 & 6 \\
\hline 5 & M & 7.5 & 2.5 & 21.9 & ALL & Standard/(-) & 2 & & 8 & & 8 & 8 & 7 \\
\hline 6 & $\mathrm{~F}$ & 7.6 & -0.2 & 15.8 & ALL & Standard $/(-)$ & 1 & 1 & 1 & 2 & 4 & 3 & 3 \\
\hline 7 & $\mathrm{~F}$ & 8.3 & -1.4 & 13.5 & ALL & Standard $/(-)$ & & & & 1 & 1 & 1 & 1 \\
\hline 8 & M & 8.4 & 0.1 & 16.4 & ALL & $\mathrm{Ph}$ positive/(-) & 2 & 1 & 3 & 3 & 5 & 5 & 3 \\
\hline 9 & M & 10.3 & -1.0 & 13.6 & LBL & $(-) /$ stage III & 1 & 1 & 1 & & 3 & 1 & 1 \\
\hline 10 & M & 10.9 & -0.6 & 15.9 & ALL & Intermediate/(-) & 1 & 1 & 1 & 2 & 3 & 4 & 3 \\
\hline 11 & M & 12.0 & -1.3 & 16.2 & ALL & Standard/(-) & 1 & 1 & 1 & & 3 & 3 & 3 \\
\hline 12 & M & 13.0 & 3.8 & 29.0 & ALL & $\mathrm{Ph}$ positive/(-) & 1 & 1 & 3 & 3 & 7 & 5 & 4 \\
\hline 13 & $\mathrm{~F}$ & 13.6 & -0.6 & 17.3 & ALL & Intermediate/(-) & 1 & 1 & 1 & 2 & 4 & 3 & 3 \\
\hline 14 & M & 15.3 & 1.0 & 24.9 & LBL & $(-) /$ stage III & 1 & 1 & 1 & 2 & 3 & 1 & 1 \\
\hline 15 & M & 16.0 & -1.8 & 17.9 & ALL & Standard $/(-)$ & & & & 1 & 1 & 1 & 1 \\
\hline 16 & $\mathrm{~F}$ & 17.8 & -0.8 & 19.7 & ALL & Intermediate/(-) & & & & 1 & 1 & 1 & 1 \\
\hline $\begin{array}{c}\text { Median } \\
\text { (IQR) }\end{array}$ & $\begin{array}{l}\text { M: } 12 \\
\text { F: } 4\end{array}$ & $\begin{array}{c}9.4 \\
(7.2 \text { to } 13.1)\end{array}$ & $\begin{array}{c}-0.6 \\
(-1.1 \text { to } 0.3)\end{array}$ & $\begin{array}{c}16.3 \\
(15.7 \text { to } 18.4)\end{array}$ & & $\begin{array}{l}\text { Number of } \\
\text { courses }\end{array}$ & 14 & 12 & 25 & 24 & 58 & 49 & 42 \\
\hline
\end{tabular}

I, induction; EC, early consolidation; C, consolidation; R, re-induction; ALL, acute lymphoblastic leukemia; Ph, Philadelphia chromosome; AKI, acute kidney injury; BMI, body mass index; Cr, creatinine; CysC, cystatin C; IQR, interquartile range; LBL, lymphoblastic lymphoma.

*Number of AKI episodes diagnosed by CysC-based eGFR; $\uparrow$ Number of AKI episodes diagnosed by Cr-based eGFR; $\$$ Number of AKI episodes diagnosed by both CysC-based eGFR and Cr-based eGFR. 
Table 2. Pediatric Risk, Injury, Failure, Loss, End Stage Renal Disease scale (pRIFLE) classification.

\begin{tabular}{lll}
\hline Category & eGFR & Urine output \\
\hline R: Risk & Decreased by $25 \%$ & $<0.5 \mathrm{~mL} / \mathrm{kg} / \mathrm{h}$ for $8 \mathrm{~h}$ \\
I: Injury & Decreased by $50 \%$ & $<0.5 \mathrm{~mL} / \mathrm{kg} / \mathrm{h}$ for $16 \mathrm{~h}$ \\
F: Failure & Decreased by $75 \%$ or $<35 \mathrm{~mL} / \mathrm{min} / 1.73 \mathrm{~m}^{2}$ & $<0.3 \mathrm{~mL} / \mathrm{kg} / \mathrm{h}$ for 24 h or anuric for $12 \mathrm{~h}$ \\
L: Loss & Loss of renal function $>4$ weeks & \\
E: End-Stage & End-stage renal disease & \\
\hline
\end{tabular}

eGFR, estimated glomerular filtration rate.

Adapted from "Akcan-Arikan, A., Zappitelli, M., Loftis, L.L., Washburn, K.K., Jefferson, L.S. \& Goldstein, S.L. (2007) Modified RIFLE criteria in critically ill children with acute kidney injury. Kidney Int., 71, 1028-1035.” with permission from International Society of Nephrology.

using the CysC- and Cr-based eGFR independently, and diagnoses were compared for AKI incidence rate, severity, and days until AKI diagnosis from the start of each treatment course.

\section{Long-term follow-up}

To assess long-term renal outcomes such as the development of CKD, CysC-based eGFR was followed until March 2021 with urine and blood tests. CKD was assessed primarily using the Kidney Disease: Improving Global Outcomes (KDIGO) definition: having either low eGFR (eGFR $<90 \mathrm{~mL} / \mathrm{min} / 1.73 \mathrm{~m}^{2}$ ) or proteinuria (protein $\geq 1+$ by urine dipstick) (Kidney Disease: Improving Global Outcomes (KDIGO) CKD Work Group 2013).

\section{Statistical methods}

Data on sex, age, type of leukemia, risk, number of courses, treatment dosage, and duration were extracted from the medical records. For data analyses, the MannWhitney $U$ test or chi-square test were used, and $p<0.05$ was considered significant. To verify the results, post hoc power analysis with the significance level $\alpha$ set to 0.05 was performed using G*Power version 3.1.9.4 (Heinrich-Heine University, Düsseldorf, Germany) (Faul et al. 2007).

\section{Results}

\section{Incidence rate and severity of AKI}

We examined 16 individuals undergoing a total of 75 courses of chemotherapy. Of these, 12 were male and the median age was 9.4 years with an interquartile range (IQR) of 7.2 to 13.1 years. The median height was $132.5 \mathrm{~cm}$ (IQR, 117.8 to $153.4 \mathrm{~cm}$ ), and the median body weight was $28.7 \mathrm{~kg}$ (IQR, 19.1 to $42.4 \mathrm{~kg}$ ). The body mass index was 16.3 (IQR, 15.7 to 18.4$) \mathrm{kg} / \mathrm{m}^{2}$. The median Z-scores for height and weight based on Japanese growth standards (Isojima et al. 2016) were -0.3 (IQR, -1.0 to 0.5 ) and -0.6 (IQR, -1.1 to 0.3 ), respectively (Table 1 ).

As shown in Table 3, all 16 patients developed AKI during chemotherapy (100\% incidence) and eventually recovered. During the 75 courses, AKI was diagnosed in 58 courses by CysC-based eGFR (77\%: "Risk" 72\%, "Injury" 4.0\%, "Failure" 1.3\%, "Loss" $0 \%$ ) and in 49 courses by Cr-based eGFR (65\%: "Risk" 61\%, "Injury"
$2.7 \%$, "Failure" 1.3\%, "Loss" 0\%). AKI was diagnosed in 42 courses by both CysC-based eGFR and Cr-based eGFR (56\%) though none developed tumor lysis syndrome during chemotherapy.

Since glucocorticoids may affect serum CysC levels (Bardi et al. 2010; Nakamura et al. 2018; Cimerman et al. 2000), the incidence of AKI based on CysC-based eGFR was compared by glucocorticoid therapy status. No significant differences were found in the incidence of AKI: $77.4 \%$ during chemotherapy with glucocorticoids and $77.3 \%$ during chemotherapy without glucocorticoids. The incidence of AKI by type of chemotherapy course is shown in Table 4 . AKI occurred frequently in consolidation courses that included methotrexate. AKI was detected in 24 of 25 consolidation courses (96\%) based on CysC-based eGFR and 22 of 25 consolidation courses (88\%) based on Cr-based eGFR (Table 4).

A discrepancy was found in 23 courses regarding the incidence of AKI diagnosed by CysC-based eGFR and Cr-based eGFR: 7 courses were diagnosed as having AKI only by Cr-based eGFR, while 16 courses were diagnosed as having AKI only by CysC-based eGFR (Table 3). However, no significant difference was observed in the incidence of AKI regardless of whether CysC-based eGFR or Cr-based eGFR was used for diagnosis $(p=0.104)$.

\section{Days until onset of AKI}

Moreover, for the 42 courses in which AKI was diagnosed by both CysC-and Cr-based eGFR, we compared the number of days from the start of each treatment course to AKI diagnosis. The median time to AKI diagnosis was significantly shorter for CysC-based eGFR ( 8 days [IQR, 4 to 21 days]) than for Cr-based eGFR (17 days [IQR, 10 to 31 days], p 0.001) (Fig. 1). Among the 42 courses in which AKI developed, there were serial changes in the reduction rate of the CysC- and Cr-based eGFR over the 2 weeks since the start of chemotherapy (Fig. 2). The CysC-based eGFR demonstrated an earlier decline than the Cr-based eGFR.

\section{Long-term follow up}

Of 16 patients, 13 were followed to March 2021. Three patients were lost to follow-up after they moved to a 
Table 3. Incidence of acute kidney injury (AKI) during the courses of chemotherapy in pediatric acute lymphoblastic leukemia/ lymphoblastic lymphoma (ALL/LBL)

\begin{tabular}{|c|c|c|c|c|c|c|c|}
\hline & & \multicolumn{2}{|c|}{ All courses } & \multicolumn{2}{|c|}{ Courses with glucocorticoids } & \multicolumn{2}{|c|}{ Courses without glucocorticoids } \\
\hline & & $\begin{array}{c}\text { CysC-based eGFR } \\
\text { based }\end{array}$ & $\begin{array}{c}\text { Cr-based eGFR } \\
\text { based }\end{array}$ & $\begin{array}{c}\text { CysC-based eGFR } \\
\text { based }\end{array}$ & $\begin{array}{c}\text { Cr-based eGFR } \\
\text { based }\end{array}$ & $\begin{array}{c}\text { CysC-based eGFR } \\
\text { based }\end{array}$ & $\begin{array}{c}\text { Cr-based eGFR } \\
\text { based }\end{array}$ \\
\hline All AKI & & $58 / 75(77.3 \%)$ & $49 / 75(65.3 \%)$ & $41 / 53(77.4 \%)$ & $34 / 53(64.2 \%)$ & $17 / 22(77.3 \%)$ & $15 / 22(68.2 \%)$ \\
\hline \multirow[t]{6}{*}{ Stage } & Risk & $54(72.0 \%)$ & $46(61.3 \%)$ & $37(69.8 \%)$ & $32(60.4 \%)$ & $17(77.3 \%)$ & $14(63.7 \%)$ \\
\hline & Injury & $3(4.0 \%)$ & $2(2.7 \%)$ & $3(5.7 \%)$ & $1(1.9 \%)$ & 0 & $1(4.5 \%)$ \\
\hline & Failure & $1(1.3 \%)$ & $1(1.3 \%)$ & $1(1.9 \%)$ & $1(1.9 \%)$ & 0 & 0 \\
\hline & Loss & 0 & 0 & 0 & 0 & 0 & 0 \\
\hline & ESRD & 0 & 0 & 0 & 0 & 0 & 0 \\
\hline & & \multicolumn{6}{|c|}{ AKI assessed by both CysC-based eGFR and Cr-based eGFR } \\
\hline \multirow[t]{2}{*}{ All AKI } & & \multicolumn{2}{|c|}{$42 / 75(56.0 \%)$} & \multicolumn{2}{|c|}{$28 / 53(52.8 \%)$} & \multicolumn{2}{|c|}{$14 / 22(63.6 \%)$} \\
\hline & & \multicolumn{6}{|c|}{ AKI assessed by CysC-based eGFR } \\
\hline \multirow[t]{2}{*}{ All AKI } & & \multicolumn{2}{|c|}{$16 / 75(21.3 \%)$} & \multicolumn{2}{|c|}{$13 / 53(24.5 \%)$} & \multicolumn{2}{|c|}{$3 / 22(13.6 \%)$} \\
\hline & & \multicolumn{6}{|c|}{ AKI assessed by Cr-based eGFR } \\
\hline All AKI & & \multicolumn{2}{|c|}{$7 / 75(9.3 \%)$} & \multicolumn{2}{|c|}{$6 / 53(11.3 \%)$} & \multicolumn{2}{|c|}{$1 / 22(4.5 \%)$} \\
\hline
\end{tabular}

AKI, acute kidney injury; CysC, cystatin C; Cr, creatinine; eGFR, estimated glomerular filtration rate; ESRD, end-stage renal disease.

Table 4. Incidence of acute kidney injury (AKI) by type of chemotherapy course and regimen in pediatric acute lymphoblastic leukemia/ lymphoblastic lymphoma (ALL/LBL).

\begin{tabular}{|c|c|c|c|c|c|c|c|c|c|}
\hline & & \multicolumn{2}{|c|}{ Induction } & \multicolumn{2}{|c|}{ Early consolidation } & \multicolumn{2}{|c|}{$\begin{array}{c}\text { Consolidation } \\
\text { (with high-dose methotrex- } \\
\text { ate) }\end{array}$} & \multicolumn{2}{|c|}{ Re-induction } \\
\hline & & $\begin{array}{l}\text { CysC-based } \\
\text { eGFR based }\end{array}$ & $\begin{array}{l}\text { Cr-based } \\
\text { eGFR based }\end{array}$ & $\begin{array}{l}\text { CysC-based } \\
\text { eGFR based }\end{array}$ & $\begin{array}{l}\text { Cr-based } \\
\text { eGFR based }\end{array}$ & $\begin{array}{l}\text { CysC-based } \\
\text { eGFR based }\end{array}$ & $\begin{array}{c}\text { Cr-based } \\
\text { eGFR based }\end{array}$ & $\begin{array}{l}\text { CysC-based } \\
\text { eGFR based }\end{array}$ & $\begin{array}{c}\text { Cr-based } \\
\text { eGFR based }\end{array}$ \\
\hline \multicolumn{2}{|c|}{ All AKI } & $8 / 14(57.1 \%)$ & $6 / 14(42.9 \%)$ & $7 / 12(58.3 \%)$ & $5 / 12(41.7 \%)$ & $24 / 25(96.0 \%)$ & $22 / 25(88.0 \%)$ & $19 / 24(79.2 \%)$ & $16 / 24(66.7 \%)$ \\
\hline \multirow[t]{6}{*}{ Stage } & Risk & $8(100 \%)$ & $6(100 \%)$ & $7(100 \%)$ & $5(100 \%)$ & $22(88.0 \%)$ & $21(84.0 \%)$ & $17(70.9 \%)$ & $14(58.4 \%)$ \\
\hline & Injury & 0 & 0 & 0 & 0 & $1(4.0 \%)$ & 0 & $2(8.3 \%)$ & $2(8.3 \%)$ \\
\hline & Failure & 0 & 0 & 0 & 0 & $1(4.0 \%)$ & $1(4.0 \%)$ & 0 & 0 \\
\hline & Loss & 0 & 0 & 0 & 0 & 0 & 0 & 0 & 0 \\
\hline & ESRD & 0 & 0 & 0 & 0 & 0 & 0 & 0 & 0 \\
\hline & & \multicolumn{8}{|c|}{ AKI assessed by both CysC-based eGFR and Cr-based eGFR } \\
\hline \multirow{2}{*}{\multicolumn{2}{|c|}{ All AKI }} & \multicolumn{2}{|c|}{$3 / 14(21.4 \%)$} & \multicolumn{2}{|c|}{$4 / 12(33.3 \%)$} & \multicolumn{2}{|c|}{$21 / 25(84.0 \%)$} & \multicolumn{2}{|c|}{$14 / 24(58.3 \%)$} \\
\hline & & \multicolumn{8}{|c|}{ AKI assessed by CysC-based eGFR } \\
\hline \multicolumn{2}{|c|}{ All AKI } & \multicolumn{2}{|c|}{$5 / 14(35.7 \%)$} & \multicolumn{2}{|c|}{$3 / 12(25.0 \%)$} & \multicolumn{2}{|c|}{$3 / 25(12.0 \%)$} & \multicolumn{2}{|c|}{$5 / 24(20.8 \%)$} \\
\hline & & \multicolumn{8}{|c|}{ AKI assessed by Cr-based eGFR } \\
\hline \multicolumn{2}{|c|}{ All AKI } & \multicolumn{2}{|c|}{$3 / 14(21.4 \%)$} & \multicolumn{2}{|c|}{$1 / 12(8.3 \%)$} & \multicolumn{2}{|c|}{$1 / 25(4.0 \%)$} & \multicolumn{2}{|c|}{$2 / 24(8.3 \%)$} \\
\hline
\end{tabular}

AKI, acute kidney injury; CysC, cystatin C; Cr, creatinine; eGFR, estimated glomerular filtration rate; ESRD, end-stage renal disease.

distant place. Follow-up duration after re-induction therapy ranged from 18 to 57 months (median, 29 months; IQR, 18-45 months). No patients presented with significant proteinuria or had CysC-based eGFR $<90 \mathrm{~mL} / \mathrm{min} / 1.73 \mathrm{~m}^{2}$.

\section{Post hoc power analysis}

A post hoc power analysis was conducted using
G*Power 3.1.9.4 (Heinrich-Heine University, Düsseldorf, Germany) to evaluate the primary outcome of time to AKI diagnosis (Faul et al. 2007). The effect size of our data was 0.609 . When the significance level $\alpha$ was set to 0.05 , the power was 0.855 , which was greater than 0.80 . Therefore, the analysis confirmed that the sample size was sufficiently large for statistical analysis (Cohen 1992). 


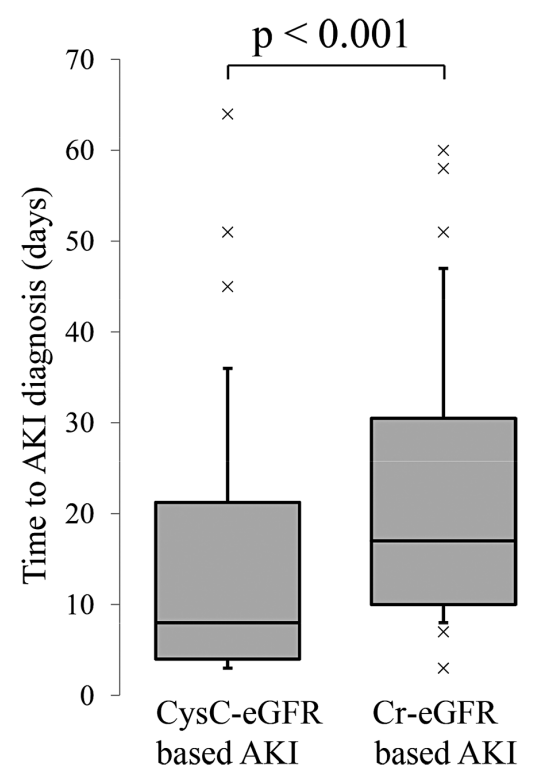

Fig. 1. Comparison of days from start of chemotherapy to acute kidney injury (AKI) diagnosis.

The median number of days to AKI $(\leq 25 \%$ of reduction in CysC-based eGFR) was 8 days (interquartile range, 4 to 21 days), compared with 17 days (interquartile range, 10 to 31 days $)$ in Cr-based eGFR $(\mathrm{p}<0.001)$. The horizontal lines in the boxes represent the median values, and the bottom and top areas of the boxes represent the 25th and 75 th percentiles, respectively. The vertical lines extend from the box to the 5th and 95th percentiles. AKI, acute kidney injury; $\mathrm{Cr}$, creatinine; CysC, cystatin $\mathrm{C}$; eGFR, estimate glomerular filtration rate.

\section{Discussion}

Our study revealed that every patient experienced AKI at least once during ALL/LBL treatment and AKI was diagnosed in $77 \%$ of the total chemotherapy courses using CysC-based eGFR. A study reported a $16.2 \%$ incidence in a cohort of 831 patients receiving chemotherapy for newonset pediatric acute myeloid leukemia (Fisher et al. 2010). Another report demonstrated a 39\% incidence of AKI in a study of 23 children with solid tumors (McMahon et al. 2018). To our knowledge, no study has evaluated the incidence of AKI among pediatric ALL/LBL patients. In the present study, the incidence of AKI during chemotherapy might be higher than the expectations of pediatric oncologists or other previously reported incidence rates in pediatric cancer patients. This discrepancy in the incidence of AKI could be explained by several reasons. First, frequent assessment of renal function under the advice of pediatric nephrologists, conducting blood tests at least 3 times weekly, led to more attentive observations, and we were able to diagnose even very mild AKI. Second, different criteria have been used in previous studies. For the diagnosis of AKI, some studies have used the Kidney Disease: Improving Global Outcomes guidelines (Sutherland et al. 2015; Meersch et al. 2017) or the pRIFLE criteria (Sethi et al. 2015; Sutherland et al. 2015), while others have used the
International Statistical Classification of Diseases and Related Health Problems codes (Ko et al. 2018). Third, the drugs used in different protocols may have influenced the results. Fourth, Cr-based eGFR was applied to assess renal function in most studies (McMahon et al. 2018), and CysCbased eGFR was rarely applied (Yong et al. 2017; Nakamura et al. 2018).

Improvements in the diagnosis and treatment of pediatric cancer patients have led to an increase in the number of cancer survivors. However, many childhood cancer survivors develop health problems years later as a result of their cancer treatment. Kooijmans et al. (2019) reviewed previous studies on renal dysfunction in childhood cancer survivors. They reported that the prevalence of CKD ranges from $2.4 \%$ to $32 \%$. Another study reported that $0.5 \%$ of over 10,000 childhood cancer survivors treated in the 1970s and 1980s had developed renal failure or required dialysis by 18 years after the initial cancer diagnosis. They had a nine-fold higher risk than their siblings without cancer (Oeffinger et al. 2006). Chemotherapy-induced AKI is characterized by several pathological states such as podocytopathy, acute tubular injury, and crystal nephropathy (Perazella 2012). Although AKI recovery, maladaptive repair, and repetitive injury in most cases could lead to tubulointerstitial fibrosis and glomerulosclerosis resulting in CKD (Venkatachalam et al. 2015; Basile et al. 2016), our findings suggest that oncologists might have overlooked mild AKIs. Cancer patients usually undergo several courses of chemotherapy, which can lead to repeated kidney injury and increased risk of CKD. Therefore, oncologists together with nephrologists must carefully detect even mild AKI during chemotherapy.

In the present study, during the 75 courses, AKI was diagnosed in 58 courses by CysC-based eGFR (77\%) and 49 courses by Cr-based eGFR (65\%). Of these cases, the diagnosis of AKI showed good agreement using the two assessment measures in 42 courses. By contrast, there was a discrepancy in the incidence of AKI diagnosed by CysCbased eGFR and Cr-based eGFR in 23 courses, although no significant difference was found in the incidence of AKI regardless of whether CysC-based eGFR or Cr-based eGFR was used for diagnosis $(p=0.104)$. We suggest the following mechanisms contribute to this discrepancy: Among the seven courses diagnosed with AKI only by Cr-based eGFR, six patients $(86 \%)$ received asparaginase immediately before the development of AKI. Since asparaginase may lower serum CysC levels by suppressing triiodothyronine blood levels, which promote CysC secretion from the smooth muscle via transforming growth factor-beta 1 , AKI evaluated using CysC-based eGFR might be overlooked because of decreased serum CysC levels in patients receiving asparaginase (Ferster et al. 1992; Kotajima et al. 2010). Meanwhile, among the 16 courses diagnosed with AKI by using the CysC-based eGFR alone, most had a negative Z-score for body weight, suggesting that they had reduced muscle mass. Since serum Cr levels are largely influenced 


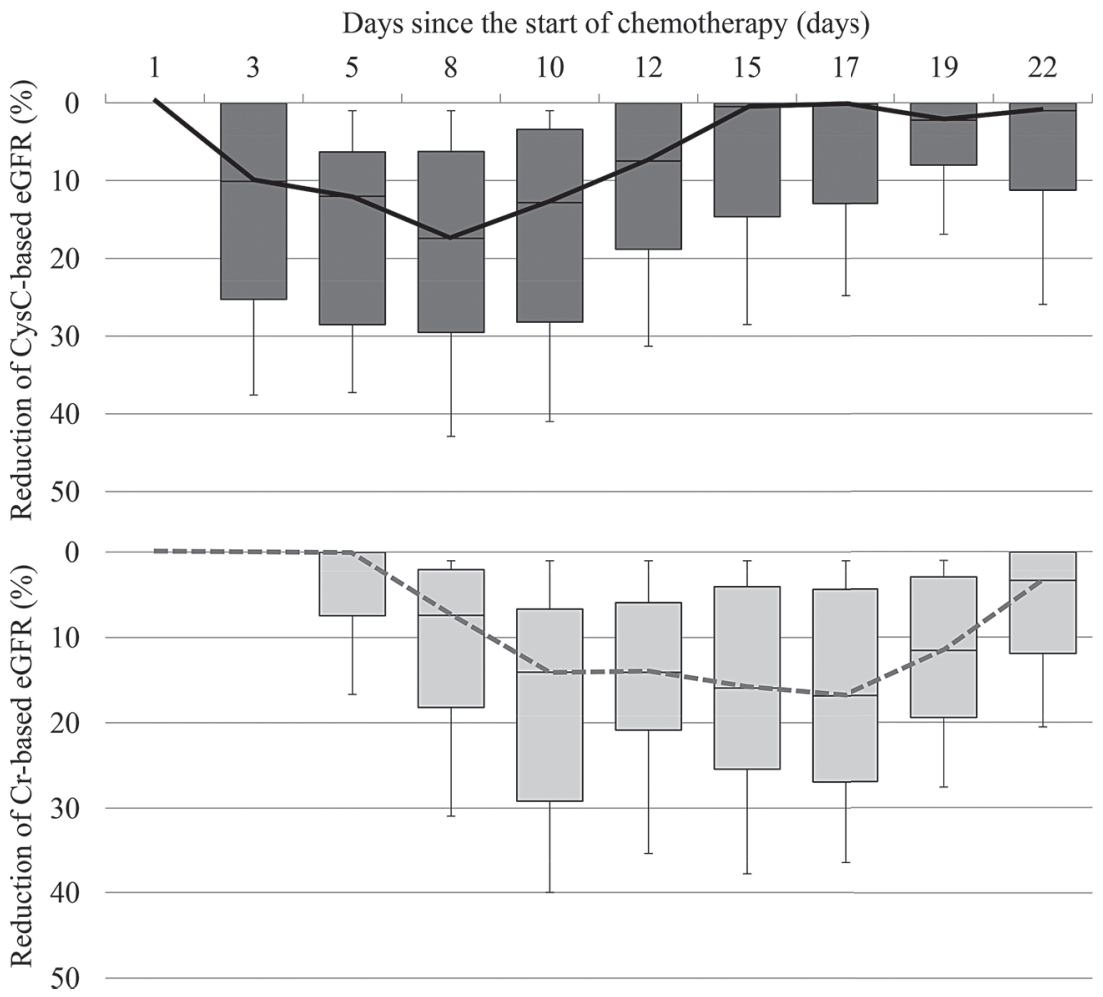

Fig. 2. Rate of CysC-based eGFR and Cr-based eGFR reduction during chemotherapy courses with acute kidney injury (AKI).

The mean reduction rate of CysC-based eGFR (solid line) and Cr-based eGFR (dashed line) since the start of chemotherapy among 42 courses with AKI is shown. The horizontal lines in the boxes represent the median values, and the bottom and top areas of the boxes represent the 25th and 75th percentiles, respectively. The vertical lines extend from the box to the 5th and 95th percentiles. AKI, acute kidney injury; Cr, creatinine; CysC, cystatin C; eGFR, estimate glomerular filtration rate.

by the muscle mass, this might have resulted in false-negative results in the diagnosis of AKI relying on Cr-based eGFR. Essentially, Rayar et al. (2013) reported that children with ALL had lower skeletal muscle mass (mean Z score of -0.18 ) than healthy children at the time of diagnosis.

Serum Cr is typically used in the diagnosis of AKI and monitoring of renal function. However, its levels may not change until approximately $50 \%$ of the kidney function has been lost (Nguyen and Devarajan 2008). CysC is a lowmolecular-weight protein constantly produced and secreted by nucleated cells throughout the body. It is freely filtered by the glomerulus and is completely reabsorbed and metabolized by the renal proximal tubules; it has an inverse correlation with GFR as measured using radioisotopes (Coll et al. 2000). Because of these biological characteristics, CysC could potentially be more suitable for the assessment of renal function in children (Lankisch et al. 2006; NakhjavanShahraki et al. 2017). A recent meta-analysis showed the superiority of CysC over Cr in diagnosing AKI (NakhjavanShahraki et al. 2017), which presents a suitable alternative to traditional diagnostic measures in nephrology. However, glucocorticoid administration in adult patients with asthma and solid tumors could increase serum CysC levels independent of renal function, even though the exact mecha- nism remains unknown (Bjarnadottir et al. 1995; Manetti et al. 2005). This may result in a falsely increased incidence of AKI if assessed by CysC-based eGFR. In contrast, there is controversy over whether glucocorticoids affect serum CysC levels in pediatric patients (Bokenkamp et al. 2002; Foster et al. 2006; Bokenkamp et al. 2007; Bardi et al. 2010; Slort et al. 2012). Therefore, in the present study, the incidence of AKI was compared separately by glucocorticoid therapy status. The results showed no significant difference between the two regimens in terms of AKI: the former was $77.4 \%$, while the latter was $77.3 \%$ (Table 3 ). Furthermore, serial changes in the rate of CysC-based eGFR reduction during 42 courses of AKI, which included 28 courses with glucocorticoids, demonstrated improvement of renal function within 12 days despite continuous glucocorticoids administration over 2 weeks. Taken together, we believe that glucocorticoids have a negligible effect on serum CysC levels and CysC-based eGFR and that the results of our study suggest that CysC-based eGFR is more sensitive than Cr-based eGFR for assessing renal function in patients with ALL/LBL. We also compared AKI incidence by type of chemotherapy course. AKI occurred often during consolidation courses, possibly due to the use of high-dose methotrexate. Although not statistically significant, in all four types of courses, AKI was 
detected more often when defined using CysC-based eGFR versus $\mathrm{Cr}$-based eGFR, which demonstrates the usefulness of CysC-based eGFR in diagnosing AKI during chemotherapy.

Although both CysC- and Cr-based eGFR have advantages and disadvantages, we found definite merit of CysC-based eGFR over Cr-based eGFR for the early diagnosis of AKI in pediatric patients with ALL/LBL undergoing chemotherapy. Detecting AKI at an early stage would allow appropriate protective measures to prevent further renal damage. Possible preventive therapeutic options including drug dosage reduction, hydration, administration of diuretics (Horie et al. 2018) and other kidney-protective drugs may contribute to the prevention of future CKD. In this study, we followed patients for a median of 29 months after re-induction courses to confirm the development of CKD; no patients had decreased eGFR. Since patients who experience $\mathrm{AKI}$ are at risk of future $\mathrm{CKD}$, we will continue further follow-up.

This study has some limitations that should be considered. First, as all study patients recovered naturally, no intervention was implemented to address AKI. Further studies are needed to determine whether recovery can be accelerated with interventions, such as dose reduction of anti-cancer agents, large-volume fluid infusion, or alkalization. Moreover, future prospective studies are required to determine whether early intervention can reduce the risk of development of CKD. Second, drugs from preceding treatment courses may have a residual effect on subsequent courses. Nevertheless, because the median CysC-based eGFR at the start of each treatment course was 160 (IQR, 135 to 181), we believe that this possibility is quite low. Third, the sample size was small. However, the sample size was sufficient for evaluating the primary outcome of time to AKI diagnosis. The effect size in our study was 0.609, which is sufficient for non-parametric data. The small sample size restricted further detailed investigations regarding the effects of individual treatment protocols or drugs. More detailed studies with more patients are necessary.

In conclusion, the results of our study highlighted two important aspects related to AKI in patients undergoing chemotherapy for pediatric ALL/LBL. First, we showed the possibility of high AKI incidence among patients. Although nephrotoxicity is well known with chemotherapy using some specific drugs, no current report has evaluated nephrotoxicity due to ALL/LBL treatment in children. Frequent observations enabled us to detect even mild AKI. Second, CysC-based eGFR allowed earlier diagnosis of AKI than Cr-based eGFR. Therefore, considering the merits and demerits of CysC-based eGFR and Cr-based eGFR in diagnosing childhood AKI during chemotherapy, we propose frequent renal function assessment by both measures.

\section{Acknowledgments}

We would like to thank Editage (https://www.editage. com) for English language editing.

\section{Author Contributions}

S.A., S.T. and K.K. designed the study; T.Y., S.A. and Y.A. collected data; T.Y., S.A., Y.A. and S.Y. analyzed data; T.Y., S.A., M.R. and Y.A. wrote the manuscript; and S.Y., T.O., T.K. and S.T. gave technical support and critically reviewed the manuscript. K.K. supervised the whole study process. All authors read and approved the final manuscript.

\section{Conflict of Interest}

The authors declare no conflict of interest.

\section{References}

Akcan-Arikan, A., Zappitelli, M., Loftis, L.L., Washburn, K.K., Jefferson, L.S. \& Goldstein, S.L. (2007) Modified RIFLE criteria in critically ill children with acute kidney injury. Kidney Int, 71, 1028-1035.

Bardi, E., Dobos, E., Kappelmayer, J. \& Kiss, C. (2010) Differential effect of corticosteroids on serum cystatin $\mathrm{C}$ in thrombocytopenic purpura and leukemia. Pathol. Oncol. Res., 16, 453-456.

Basile, D.P., Bonventre, J.V., Mehta, R., Nangaku, M., Unwin, R., Rosner, M.H., Kellum, J.A. \& Ronco, C.; ADQI XIII Work Group (2016) Progression after AKI: understanding maladaptive repair processes to predict and identify therapeutic treatments. J. Am. Soc. Nephrol., 27, 687-697.

Bjarnadottir, M., Grubb, A. \& Olafsson, I. (1995) Promoter-mediated, dexamethasone-induced increase in cystatin C production by HeLa cells. Scand. J. Clin. Lab. Invest., 55, 617-623.

Bokenkamp, A., Laarman, C.A., Braam, K.I., van Wijk, J.A., Kors, W.A., Kool, M., de Valk, J., Bouman, A.A., Spreeuwenberg, M.D. \& Stoffel-Wagner, B. (2007) Effect of corticosteroid therapy on low-molecular weight protein markers of kidney function. Clin. Chem., 53, 2219-2221.

Bokenkamp, A., van Wijk, J.A., Lentze, M.J. \& Stoffel-Wagner, B. (2002) Effect of corticosteroid therapy on serum cystatin C and beta2-microglobulin concentrations. Clin. Chem., 48, 1123-1126.

Ciarimboli, G., Holle, S.K., Vollenbrocker, B., Hagos, Y., Reuter, S., Burckhardt, G., Bierer, S., Herrmann, E., Pavenstadt, H., Rossi, R., Kleta, R. \& Schlatter, E. (2011) New clues for nephrotoxicity induced by ifosfamide: preferential renal uptake via the human organic cation transporter 2. Mol. Pharm., 8, 270-279.

Cimerman, N., Brguljan, P.M., Krasovec, M., Suskovic, S. \& Kos, J. (2000) Serum cystatin C, a potent inhibitor of cysteine proteinases, is elevated in asthmatic patients. Clin. Chim. Acta, 300, 83-95.

Cohen, J. (1992) A power primer. Psychol. Bull., 112, 155-159.

Coll, E., Botey, A., Alvarez, L., Poch, E., Quinto, L., Saurina, A., Vera, M., Piera, C. \& Darnell, A. (2000) Serum cystatin C as a new marker for noninvasive estimation of glomerular filtration rate and as a marker for early renal impairment. Am. J. Kidney Dis., 36, 29-34.

Faul, F., Erdfelder, E., Lang, A.G. \& Buchner, A. (2007) G*Power 3: a flexible statistical power analysis program for the social, behavioral, and biomedical sciences. Behav. Res. Methods, 39, 175-191.

Ferster, A., Glinoer, D., Van Vliet, G. \& Otten, J. (1992) Thyroid function during L-asparaginase therapy in children with acute lymphoblastic leukemia: difference between induction and late intensification. Am. J. Pediatr. Hematol. Oncol., 14, 192-196.

Fisher, B.T., Zaoutis, T.E., Leckerman, K.H., Localio, R. \& Aplenc, 
R. (2010) Risk factors for renal failure in pediatric patients with acute myeloid leukemia: a retrospective cohort study. Pediatr. Blood Cancer, 55, 655-661.

Foster, J., Reisman, W., Lepage, N. \& Filler, G. (2006) Influence of commonly used drugs on the accuracy of cystatin C-derived glomerular filtration rate. Pediatr. Nephrol., 21, 235-238.

Horie, S., Oya, M., Nangaku, M., Yasuda, Y., Komatsu, Y., Yanagita, M., Kitagawa, Y., Kuwano, H., Nishiyama, H., Ishioka, C., Takaishi, H., Shimodaira, H., Mogi, A., Ando, Y., Matsumoto, K., et al. (2018) Guidelines for treatment of renal injury during cancer chemotherapy 2016. Clin. Exp. Nephrol., 22, 210-244.

Isojima, T., Kato, N., Ito, Y., Kanzaki, S. \& Murata, M. (2016) Growth standard charts for Japanese children with mean and standard deviation (SD) values based on the year 2000 national survey. Clin. Pediatr. Endocrinol., 25, 71-76.

Kellum, J.A. \& Lameire, N.; KDIGO AKI Guideline Work Group (2013) Diagnosis, evaluation, and management of acute kidney injury: a KDIGO summary (Part 1). Crit. Care, 17, 204.

Kidney Disease: Improving Global Outcomes (KDIGO) CKD Work Group (2013) KDIGO 2012 clinical practice guideline for the evaluation and management of chronic kidney disease. Kidney Inter. Suppl., 3, 1-150.

Ko, S., Venkatesan, S., Nand, K., Levidiotis, V., Nelson, C. \& Janus, E. (2018) International statistical classification of diseases and related health problems coding underestimates the incidence and prevalence of acute kidney injury and chronic kidney disease in general medical patients. Intern. Med. J., 48, 310-315.

Kooijmans, E.C., Bokenkamp, A., Tjahjadi, N.S., Tettero, J.M., van Dulmen-den Broeder, E., van der Pal, H.J. \& Veening, M.A. (2019) Early and late adverse renal effects after potentially nephrotoxic treatment for childhood cancer. Cochrane Database Syst. Rev., 3, CD008944.

Kotajima, N., Yanagawa, Y., Aoki, T., Tsunekawa, K., Morimura, T., Ogiwara, T., Nara, M. \& Murakami, M. (2010) Influence of thyroid hormones and transforming growth factor-beta1 on cystatin C concentrations. J. Int. Med. Res., 38, 1365-1373.

Lahoti, A., Kantarjian, H., Salahudeen, A.K., Ravandi, F., Cortes, J.E., Faderl, S., O’Brien, S., Wierda, W. \& Mattiuzzi, G.N. (2010) Predictors and outcome of acute kidney injury in patients with acute myelogenous leukemia or high-risk myelodysplastic syndrome. Cancer, 116, 4063-4068.

Lankisch, P., Wessalowski, R., Maisonneuve, P., Haghgu, M., Hermsen, D. \& Kramm, C.M. (2006) Serum cystatin C is a suitable marker for routine monitoring of renal function in pediatric cancer patients, especially of very young age. Pediatr. Blood Cancer, 46, 767-772.

Laterza, O.F., Price, C.P. \& Scott, M.G. (2002) Cystatin C: an improved estimator of glomerular filtration rate? Clin. Chem., 48, 699-707.

Manetti, L., Genovesi, M., Pardini, E., Grasso, L., Lupi, I., Linda Morselli, L., Pellegrini, G. \& Martino, E. (2005) Early effects of methylprednisolone infusion on serum cystatin $\mathrm{C}$ in patients with severe Graves' ophthalmopathy. Clin. Chim. Acta, 356, 227-228.

McMahon, K.R., Harel-Sterling, M., Pizzi, M., Huynh, L., Hessey, E. \& Zappitelli, M. (2018) Long-term renal follow-up of children treated with cisplatin, carboplatin, or ifosfamide: a pilot study. Pediatr. Nephrol., 33, 2311-2320.

Meersch, M., Schmidt, C., Hoffmeier, A., Van Aken, H., Wempe,
C., Gerss, J. \& Zarbock, A. (2017) Prevention of cardiac surgery-associated AKI by implementing the KDIGO guidelines in high risk patients identified by biomarkers: the PrevAKI randomized controlled trial. Intensive Care Med., 43, 1551-1561.

Nakamura, N., Watanabe, H., Okamura, K. \& Kagami, S. (2018) Assessment of renal function in Japanese children with malignancies using serum cystatin C. J. Med. Invest., 65, 231-235.

Nakhjavan-Shahraki, B., Yousefifard, M., Ataei, N., Baikpour, M., Ataei, F., Bazargani, B., Abbasi, A., Ghelichkhani, P., Javidilarijani, F. \& Hosseini, M. (2017) Accuracy of cystatin C in prediction of acute kidney injury in children; serum or urine levels: which one works better? A systematic review and metaanalysis. BMC Nephrol., 18, 120.

Nguyen, M.T. \& Devarajan, P. (2008) Biomarkers for the early detection of acute kidney injury. Pediatr. Nephrol., 23, 21512157.

Oeffinger, K.C., Mertens, A.C., Sklar, C.A., Kawashima, T., Hudson, M.M., Meadows, A.T., Friedman, D.L., Marina, N., Hobbie, W., Kadan-Lottick, N.S., Schwartz, C.L., Leisenring, W. \& Robison, L.L.; Childhood Cancer Survivor Study Group (2006) Chronic health conditions in adult survivors of childhood cancer. N. Engl. J. Med., 355, 1572-1582.

Pabla, N. \& Dong, Z. (2008) Cisplatin nephrotoxicity: mechanisms and renoprotective strategies. Kidney Int., 73, 994-1007.

Perazella, M.A. (1999) Crystal-induced acute renal failure. Am. J. Med., 106, 459-465.

Perazella, M.A. (2012) Onco-nephrology: renal toxicities of chemotherapeutic agents. Clin. J. Am. Soc. Nephrol., 7, 17131721.

Rayar, M., Webber, C.E., Nayiager, T., Sala, A. \& Barr, R.D. (2013) Sarcopenia in children with acute lymphoblastic leukemia. J. Pediatr. Hematol. Oncol., 35, 98-102.

Sethi, S.K., Kumar, M., Sharma, R., Bazaz, S. \& Kher, V. (2015) Acute kidney injury in children after cardiopulmonary bypass: risk factors and outcome. Indian Pediatr., 52, 223-226.

Slort, P.R., Ozden, N., Pape, L., Offner, G., Tromp, W.F., Wilhelm, A.J. \& Bokenkamp, A. (2012) Comparing cystatin C and creatinine in the diagnosis of pediatric acute renal allograft dysfunction. Pediatr. Nephrol., 27, 843-849.

Sutherland, S.M., Byrnes, J.J., Kothari, M., Longhurst, C.A., Dutta, S., Garcia, P. \& Goldstein, S.L. (2015) AKI in hospitalized children: comparing the pRIFLE, AKIN, and KDIGO definitions. Clin. J. Am. Soc. Nephrol., 10, 554-561.

Uemura, O., Nagai, T., Ishikura, K., Ito, S., Hataya, H., Gotoh, Y., Fujita, N., Akioka, Y., Kaneko, T. \& Honda, M. (2014a) Creatinine-based equation to estimate the glomerular filtration rate in Japanese children and adolescents with chronic kidney disease. Clin. Exp. Nephrol., 18, 626-633.

Uemura, O., Nagai, T., Ishikura, K., Ito, S., Hataya, H., Gotoh, Y., Fujita, N., Akioka, Y., Kaneko, T. \& Honda, M. (2014b) Cystatin C-based equation for estimating glomerular filtration rate in Japanese children and adolescents. Clin. Exp. Nephrol., 18, 718-725.

Venkatachalam, M.A., Weinberg, J.M., Kriz, W. \& Bidani, A.K. (2015) Failed tubule recovery, AKI-CKD transition, and kidney disease progression. J. Am. Soc. Nephrol., 26, 17651776.

Yong, Z., Pei, X., Zhu, B., Yuan, H. \& Zhao, W. (2017) Predictive value of serum cystatin $\mathrm{C}$ for acute kidney injury in adults: a meta-analysis of prospective cohort trials. Sci. Rep., 7, 41012. 